\title{
Memory effect in cadmium telluride quantum dots doped ferroelectric liquid crystals
}

\author{
A. Kumar, ${ }^{1}$ J. Prakash, ${ }^{2}$ Mohd Taukeer Khan, ${ }^{3}$ S. K. Dhawan, ${ }^{3}$ and A. M. Biradar ${ }^{1, a)}$ \\ ${ }^{1}$ Liquid Crystal Group, National Physical Laboratory, Dr. K. S. Krishnan Road, New Delhi 110012, India \\ ${ }^{2}$ Instrument Design Development Centre, Indian Institute of Technology Delhi, Hauz Khas, \\ New Delhi 110016, India \\ ${ }^{3}$ Conducting Polymer Group, National Physical Laboratory, Dr. K. S. Krishnan Road, \\ New Delhi 110012, India
}

(Received 17 August 2010; accepted 5 September 2010; published online 21 October 2010)

\begin{abstract}
A pronounced memory effect has been observed in cadmium telluride quantum dots (CdTe-QDs) doped ferroelectric liquid crystals (FLCs) by using dielectric and electro-optical methods. The memory effect has been attributed to the charge storage on the CdTe-QDs upon the application of dc bias across the sample cell. The FLC molecules remain in the switched state in vicinity of the charge stored on QDs even after removal of bias. It has been observed that the memory effect depends on doping concentrations of CdTe-QDs and the FLC material used. (C) 2010 American Institute of Physics. [doi:10.1063/1.3495780]
\end{abstract}

Ferroelectric liquid crystals (FLCs) have been employed in various promising applications, such as flat panel displays, spatial light modulators, optical antennas, etc., due to their important characteristic features such as good optical contrast, fast response, low threshold voltage, and memory effect. The metal nanoparticles (NPs) have been doped into liquid crystals (LCs) to observe various interesting phenomena, such as nonvolatile memory effect, ${ }^{1}$ enhanced photoluminescence, ${ }^{2}$ enhanced electrical conductivity, ${ }^{3}$ and induced LC alignments. ${ }^{4}$ The doping of NPs has improved the electro-optical properties such as good contrast and low power operation of LC based display devices. ${ }^{5,6}$ The semiconducting quantum dots (QDs) have attracted a great deal of interest in the scientific community for their promising applications such as next generation photonic devices, QD displays, and biomedical imaging. ${ }^{7-10}$ Apart from them, various studies have been carried out by researchers for utilizing the QDs in the realization of nonvolatile memory devices. Nassiopoulou et al. ${ }^{11}$ have observed a large shift in the capacitance-voltage $(\mathrm{C}-\mathrm{V})$ hysteresis of the metal-oxidesemiconductor (MOS) structures containing germanium QDs. The memory effect has been observed in a MOS structure containing zirconium $(\mathrm{Zr})$ nanocrystals embedded in $\mathrm{ZrO}_{2}$ dielectric layer by Lee et al. ${ }^{12}$ The high frequency $\mathrm{C}-\mathrm{V}$ characteristics of the structures with silicon NPs have shown hysteresis indicating the charging of NPs with electrons/ holes due to charge carrier tunneling from the substrate through the thin oxide at positive/negative biases. ${ }^{13}$ Charge retention properties of QDs have been studied ${ }^{14,15}$ and found that the charge carriers stored on QDs could persist over time scales exceeding seconds or even hours. ${ }^{16}$

On the other hand, the anisotropic ordering of LCs can impart order onto the nanosized guest particles and hence researchers have utilized this property to organize QDs in the form of their self-assembly. ${ }^{17}$ Hirst et al. ${ }^{18}$ studied the mechanism to organize QDs by using anisotropic LC medium and explored the possibility for the fabrication of mul-

\footnotetext{
${ }^{\text {a) }}$ Author to whom correspondence should be addressed. Electronic mail: abiradar@mail.nplindia.ernet.in.
}

tifunctional switchable devices. However, the effect of QDs on the properties of LC materials is rarely reported. The understanding of the interaction between QDs and LCs is a challenging area of research for its utilization to achieve controlled self-assembly of QDs and improved electro-optical characteristics of LCs.

In this paper, we observed the effect of cadmium telluride (CdTe)-QDs on the electro-optical properties of FLCs. It has been observed that the doping of CdTe-QDs in various FLC materials favors pronounced memory effect. The observed memory state does not come back to its original (unswitched) state instantaneously but it retains in that state for a remarkable time. We also observed that retention of memory state depends on the doping concentration of QDs and FLC materials.

The CdTe-QDs have been synthesized in the form of P3HT (Poly-3(hexylthiophene))-CdTe nanocomposites. The P3HT polymer is used for capping to prevent the agglomeration of QDs. The high resolution transmission electron microscopy (HRTEM) images and electron diffraction pattern of the synthesized P3HT-CdTe nanocomposites have been shown in Fig. 1. Highly resolved HRTEM image has also been given in the inset of Fig. 1(a). The typical size of CdTeQDs varies between 2-7 nm in diameter. The sample cells for the present study were prepared using indium tin oxide coated glass plates. The desired (squared) electrode area was $0.45 \times 0.45 \mathrm{~cm}^{2}$. The thickness of the cell was maintained by using $\sim 4 \mu \mathrm{m}$ thick Mylar spacers. The FLC materials

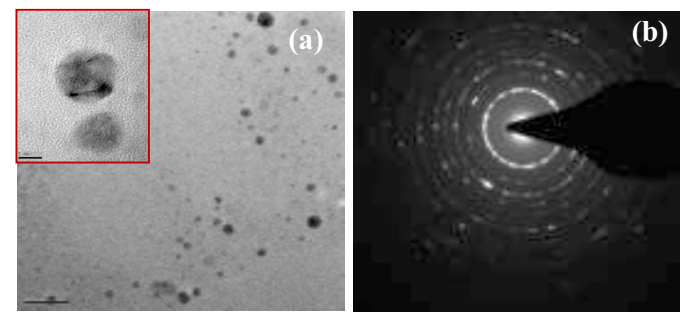

FIG. 1. (Color online) (a) HRTEM image with scale bar: $20 \mathrm{~nm}$ (the inset shows highly magnified image with scale bar: $5 \mathrm{~nm}$ (b) electron diffraction pattern of P3HT/CdTe-QDs nanocomposites. 


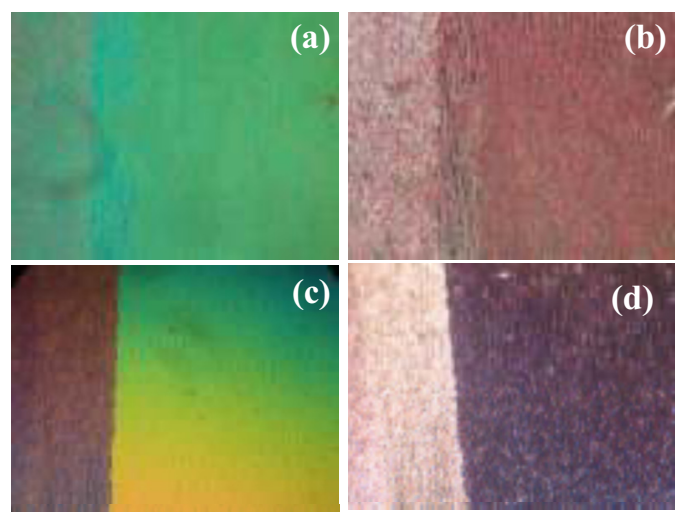

FIG. 2. (Color online) Polarizing optical micrographs of (a) bright state, (b) dark state of pure and (c) bright state, (d) dark state of $\sim 4$ wt \% CdTe-QDs doped LAHS19 material.

(pure and CdTe-QDs doped) were filled into these cells by capillary action above their respective isotropic temperatures. The homogeneous alignment of FLC cells has been achieved by using rubbed polyimide technique. The phase sequences of FLC materials used are as follows:

$$
\begin{aligned}
& 7{ }^{\circ} \mathrm{C} \quad 59.5^{\circ} \mathrm{C} \quad 60^{\circ} \mathrm{C} \\
& \text { cryt. } \leftrightarrow S^{*} C^{*} \leftrightarrow S m A^{*} \leftrightarrow \text { iso. (LAHS19) } \\
& 1{ }^{\circ} \mathrm{C} \quad 58{ }^{\circ} \mathrm{C} \quad 64^{\circ} \mathrm{C} \\
& \text { cryt. } \leftrightarrow \text { SmC }^{*} \leftrightarrow \text { SmA }^{*} \leftrightarrow \text { iso. (LAHS18) } \\
& -14{ }^{\circ} \mathrm{C} \quad 60.5^{\circ} \mathrm{C} \quad 64^{\circ} \mathrm{C} \\
& \text { cryt. } \leftrightarrow S m C^{*} \leftrightarrow S m A^{*} \leftrightarrow \text { iso. (FLC 6304) }
\end{aligned}
$$

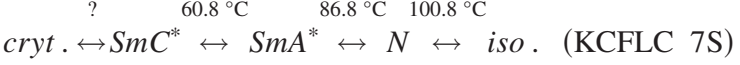

The dielectric measurements of pure and CdTe-QDs doped FLC materials were performed using an impedance analyzer (Wayne Kerr, 6540A, U.K. ) in the frequency range $20 \mathrm{~Hz}-1$ $\mathrm{MHz}$ with measuring voltage of $0.5 \mathrm{~V}$. The optical micrographs were taken using polarizing optical microscope (Carl Zeiss, Germany).

Figure 2 shows the optical micrographs of bright and dark states of pure and $\sim 4$ wt \% CdTe-QDs doped FLC (LAHS19). It can be seen clearly from Fig. 2, that the doping of CdTe-QDs does not perturb the alignment of LAHS19 material remarkably. However, the presence of CdTe-QDs in the material can be clearly seen in the form of light scattering centers in the dark state of CdTe-QDs doped LAHS19 [Fig. 2(d)]. It is found that the dispersion of spherical particles such as cadmium sulphide QDs into the LCs typically introduce random surfaces that can disrupt the uniform LC alignment. ${ }^{17}$ It has also been observed that the presence of large colloidal particles in LCs is usually associated with a defect in the texture. ${ }^{18}$ The memory effect in CdTe-QDs doped various FLC material has been observed using dielectric measurements. Figure 3 shows the behavior of dielectric permittivity $\left(\varepsilon^{\prime}\right)$ with frequency at different dc biases of CdTe-QDs doped FLCs. The behavior of memory in pure FLC materials has been shown in insets of figures. All FLC materials used exhibit $\mathrm{SmC}^{*}$ phase at room temperature. In $\mathrm{SmC}^{*}$ phase, the FLC molecules are arranged in a helicoidal manner and show higher value of $\varepsilon^{\prime}$ due to the contribution of Goldstone mode (GM) which occurs due to phase fluctuation of FLC director. The application of sufficient bias across the cell suppresses the GM contribution (due to unwinding of
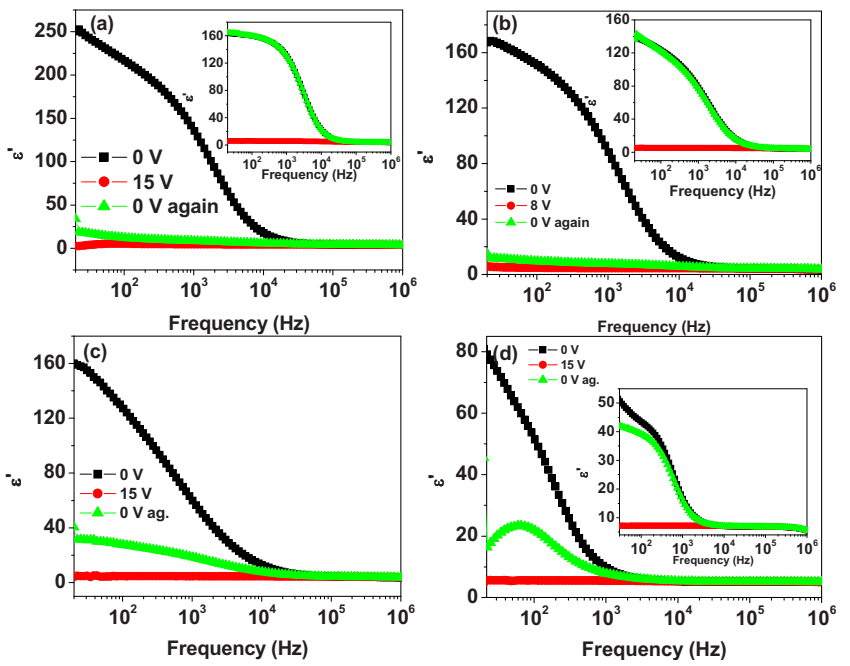

FIG. 3. (Color online) Behavior of dielectric permittivity $\left(\varepsilon^{\prime}\right)$ at different dc biases of CdTe-QDs doped (a) LAHS19, (b) LAHS18, and (c) FLC 6304 in $\sim 4 \mu \mathrm{m}$ thick cells, and (d) KCFLC $7 \mathrm{~S}$ in $\sim 10 \mu \mathrm{m}$ thick cell at room temperature.

helicoidal structure) and results in the lower and static value of $\varepsilon^{\prime}$. On the removal of bias, FLC molecules attain their helicoidal structure again due to restoring forces and $\varepsilon^{\prime}$ reaches its original value. If somehow, FLC molecules retain their switched state (unwound state) even after removal of bias then it means the material exhibits memory. Figure 3(a) shows the occurrence of dielectric memory in CdTe-QDs doped LAHS19 material. It can be seen clearly from figure that the switched state has been retained for CdTe-QDs doped LAHS19 even after removal of bias whereas it restored its original state instantaneously in case of pure LAHS19 [inset of Fig. 3(a)]. Similarly, Figs. 3(b)-3(d) depict the occurrence of dielectric memory in CdTe-QDs doped LAHS18, FLC6304, and KCFLC 7S materials, respectively. It is evident from Figs. 3(b)-3(d) that all FLC materials show dielectric memory by doping CdTe-QDs whereas pure counterparts do not show the same as can be seen from the insets [of Figs. 3(b) and 3(d)]. The memory effect in pure FLC 6304 has been studied and found that it does not show memory effect by dielectric measurements. ${ }^{1}$ It has been demonstrated earlier that surface stabilization (SS) leads to the bistability and memory effect in FLCs. ${ }^{19}$ To exclude the possibility of SS, we used either DHFLC material (which have ultrashort pitch) or FLC material keeping cell thickness greater than pitch value. This means that by doping CdTeQDs in the conventional FLCs, one can get a memory state even in the thicker non-SS FLC geometries [Fig. 3(d)].

It has been observed that the dielectric memory persisted over time scales exceeding minutes. The value of $\varepsilon^{\prime} \mathrm{CdTe}-$ QDs doped LAHS19 material does not reach its original $(0 \mathrm{~V})$ value instantaneously but takes some remarkable time. The value of $\varepsilon^{\prime}$ after 4 min was found almost $50 \%$ of its original value indicating that almost half of the FLC molecules were still in switched state even after 4 min of removal of bias. The retention of memory state for other FLCs/ CdTe-QDs composites has also been observed. It has been found that the retention of memory state follows the same trend in case of FLCs/CdTe-QDs as CdTe-QDs doped LAHS19. However, the retention time is found to be dependent on the concentration of CdTe-QDs and the FLC materials used. 
The occurrence of memory effect can be understood by taking the interaction of FLC molecules with CdTe-QDs into account. The operation of a memory device in a junctionlike CdS nanocomposites/conducting polymer poly[2-methoxy-5(2-ethylhexyloxy)-1,4-phenylene-vinylene] heterostructure embedded in polyvinyl alcohol (PVA) matrix has been demonstrated by studying $\mathrm{C}-\mathrm{V}$ characteristics, and attributed this memory behavior to the trapping, storage, and emission of holes in the CdS nanoneedles embedded in PVA matrix. ${ }^{20}$ The phenomena such as QD memory ${ }^{21}$ and electrical bistability $^{22}$ in CdSe-QDs based devices due to their charge trapping and storage characteristics have also been demonstrated. The CdTe-QDs used for this study have been grown in situ in the matrix of conducting polymer $\mathrm{P} 3 \mathrm{HT}$, i.e., the CdTe-QDs are capped with P3HT. The applications of dc bias across the CdTe-QDs doped FLCs sample cells induce charge transfer from FLC molecules to CdTe-QDs. The $\mathrm{P} 3 \mathrm{HT}$ is a conducting polymer and hence it provided a low resistive path to the charges in reaching toward CdTe-QDs. The charge stored on CdTe-QDs can retain for several minutes and hence it does not remove instantaneously after removal of bias. The FLC molecules nearby these CdTe-QDs retain their switched state in the effect of charge stored on the CdTe-QDs which has been resulted in the form of pronounced dielectric memory. It is found that the charge stored on the CdTe-QDs has been released in a slower fashion and taken upto 5 min duration to discharge completely in case of $\sim 4$ wt \% CdTe-QDs doped LAHS19 material. Moreover, the charge retention time on CdTe-QDs is found to depend on the FLC material used. The maximum charge retention time of $\sim 10 \mathrm{~min}$ has been observed in case of $\sim 4$ wt $\%$ CdTe-QDs doped LAHS18 material which has slightly different composition than that of LAHS19 whereas in case of KCFLC 7S/CdTe-QDs composite, $\varepsilon^{\prime}$ reached its original value $(0 \mathrm{~V}$ state $)$ in comparatively shorter duration ( $\sim 1 \mathrm{~min})$. However, to understand the exact nature of interactions between FLC molecules and QDs in achieving improved electro-optical properties of FLCs are still a challenging task to be investigated.

A pronounced memory effect in FLCs/CdTe-QDs composites has been observed. The observed memory effect has been attributed to conducting polymer mediated charge transfer from FLC molecules to CdTe-QDs. It is found that the stored charges on CdTe-QDs do not remove instantaneously after removal of bias but they take several minutes to discharge completely. The retention of memory state is found to be dependent on the concentration of CdTe-QDs and the FLC material used.

The authors sincerely thank Professor R. C. Budhani, the Director, National Physical Laboratory, for continuous encouragement and interest in this work. We sincerely thank Dr. Poonam Silotia of Delhi University for useful discussions. The authors (A.K. and J.P.) are thankful to University Grant Commission (UGC) and Council of Scientific and Industrial Research (CSIR), New Delhi for providing financial assistance.

${ }^{1}$ J. Prakash, A. Choudhary, A. Kumar, D. S. Mehta, and A. M. Biradar, Appl. Phys. Lett. 93, 112904 (2008).

${ }^{2}$ A. Kumar, J. Prakash, D. S. Mehta, W. Haase, and A. M. Biradar, Appl. Phys. Lett. 95, 023117 (2009).

${ }^{3}$ L. A. Holt, B. J. Richard, E. D. Stephen, B. Andrew, and S. Gordon, J. Appl. Phys. 103, 063712 (2008).

${ }^{4}$ H. Qi and T. Hegmann, Appl. Mater. Inter 1, 1731 (2009).

${ }^{5}$ T. Joshi, A. Kumar, J. Prakash, and A. M. Biradar, Appl. Phys. Lett. 96, 253109 (2010)

${ }^{6}$ W.-K. Lee, J.-H. Choi, H.-J. Na, J.-H. Lim, J.-M. Han, J.-Y. Hwang, and D.-S. Seo, Opt. Lett. 34, 3653 (2009).

${ }^{7}$ X. Tong and Y. Zhao, J. Am. Chem. Soc. 129, 6372 (2007).

${ }^{8}$ S. W. Lee, C. Mao, C. E. Flynn, and A. M. Belcher, Science 296, 892 (2002).

${ }^{9}$ A. Balandin, K. L. Wang, N. Kouklin, and S. Bandyopadhyay, Appl. Phys. Lett. 76, 137 (2000).

${ }^{10}$ M. Tamborra, M. Striccoli, R. Comparelli, M. Curri, A. Petrella, and A. Agestiano, Nanotechnology 15, S240 (2004).

${ }^{11}$ A. G. Nassiopoulou, A. Olzierski, E. Tsoi, I. Berbezier, and A. Karmous, J. Nanosci. Nanotechnol. 7, 316 (2007).

${ }^{12}$ J. H. Lee, J. S. Choi, S. Hong, I. Hwang, Y.-I. Kim, S. J. Ahn, S.-O. Kang, and B. H. Park, Jpn. J. Appl. Phys. 46, L1246 (2007).

${ }^{13}$ N. Nedev, D. Nesheva, E. Manolov, R. Bruggemann, S. Meier, and Z. Levi, Proceedings of the 26th International Conference on Microelectronics, 11-14 May, pp. 117-120 (2008).

${ }^{14}$ T. Lundstrom, W. Schoenfeld, H. Lee, and P. M. Petroff, Science 286, 2312 (1999).

${ }^{15}$ J. J. Finley, M. Skalitz, M. Arzberger, A. Zrenner, G. Bohm, and G. Abstreiter, Appl. Phys. Lett. 73, 2618 (1998).

${ }^{16}$ D. Nesheva, N. Nedev, E. Manolov, I. Bineva, and H. Hofmeister, J. Phys. Chem. Solids 68, 725 (2007).

${ }^{17}$ R. Basu and G. S. Iannacchione, Phys. Rev. E 80, 010701(R) (2009).

${ }^{18}$ L. S. Hirst, J. Kirchhoff, R. Inman, and S. Ghosh, Proc. SPIE 7618, 76180F (2010).

${ }^{19}$ N. A. Clark and S. T. Lagerwall, Appl. Phys. Lett. 36, 899 (1980).

${ }^{20}$ S. P. Mondal, V. S. Reddy, S. Das, A. Dhar, and S. K. Ray, Nanotechnology 19, 215306 (2008).

${ }^{21}$ M. D. Fischbein and M. Drndic, Appl. Phys. Lett. 86, 193106 (2005).

${ }^{22}$ K. Mohanta, S. K. Majee, S. K. Batabyal, and A. J. Pal, J. Phys. Chem. B 110, 18231 (2006). 